\title{
Photopolymerization of Methyl Methacrylate with $N$-Benzylpyridinium Bromide
}

\author{
Kenzo TABUCHI and Naokazu SAKOTA* \\ Department of Industrial Chemistry, Niihama Technical College, \\ 7-1 Yagumo-cho, Niihama 792, Japan \\ *Sugai Chemical Co., Ltd., 4-4-6 Uzu, Wakayama 641, Japan
}

(Received July 15, 1982)

\begin{abstract}
Photopolymerization of methyl methacrylate (MMA) with $N$-benzylpyridinium bromide (BPB) was investigated in three different solvents, methanol, acetonitrile and methylene chloride. Electronic spectra and electric conductivity were also measured in the polymerization systems. The results indicated that BPB existed in an equilibrium among free ions, an ion pair, and $\mathrm{CT}$ complex in the polymerization system, and that the equilibrium shifted to the free ions in polar solvent and to the CT complex in a less polar solvent. Kinetic studies of the photopolymerization of MMA with BPB suggest that this is probably initiated by a bromine atom formed by the photodecomposition of the CT complex of BPB. In methylene chloride the photopolymerization of MMA with BPB yielded an oligomer (OMMA, $M_{n}=650$ ). The structural analysis of the OMMA showed that it had both a bromine atom and a dihydropyridine group per each molecule.

KEY WORDS Photopolymerization / $N$-Benzylpyridinium Bromide / Charge-Transfer Complex / Oligo(methyl methacrylate) / Hydropyridine /
\end{abstract}

The authors reported that bromine acted as a photosensitizer for the polymerization of vinyl monomers, such as methyl methacrylate (MMA), styrene (St), acrylonitrile (AN), and ethyl acrylate (EA), and that the photopolymerization of MMA was significantly accelerated by addition of pyridine. ${ }^{1}$ The acceleration effect by pyridine was suggested to be due to the pyridine-bromine complex $\left(\mathrm{Py}-\mathrm{Br}_{2}\right)$ formed as an intermediate in the polymerization system. The authors also clarified that, even in the dark, the $\mathrm{Py}-\mathrm{Br}_{2}$ reacted readily with MMA to form both a crystalline material and dibrominated methyl methacrylate (DBMMA), and that the crystalline material acted as a more effective photosensitizer than DBMMA for the photopolymerization of vinyl monomers. ${ }^{2}$

Later, the authors showed that the crystalline material described above consisted of equimolar amounts of MMA, pyridine and bromine, and was identified to be methyl $\alpha$-bromo- $\alpha$-methyl- $\beta$ pyridiniopropionate bromide (BPPB). ${ }^{3}$ In the same way acrylonitrile was found to react with $\mathrm{Py}_{-} \mathrm{Br}_{2}$ to form $\alpha$-bromo- $\beta$-pyridinioproponitrile bromide (BPNB).
In the present paper, the authors have studied the photopolymerization of MMA with $N$-benzylpyridinium bromide (BPB) which acts as a photosensitizer, as well as other $N$-substituted pyridinium bromides, such as BPPB and BPNB. The photopolymerization of MMA with BPB in methylene chloride, however, merely gave a poly(methyl methacrylate) (PMMA) of relatively low molecular weight. The profile of the photopolymerization is given in this paper along with kinetic studies on the photopolymerization and analysis of oligo(methyl methacrylate) (OMMA).

\section{EXPERIMENTAL}

\section{Materials}

MMA and St were purified by the usual methods immediately before use. BPB was prepared by the Menschutkin reaction. Triethylammonium bromide (TEAB) was prepared by leading hydrogen bromide gas into an ethanolic solution of triethylamine. Methylene chloride, acetonitrile and methanol were purified by the usual methods. 


\section{Electronic Spectra}

Electronic spectra were recorded on a Japan Spectroscopic Company Model UVIDEC-1 spectrophotometer and were measured in a quartz cell of $10 \mathrm{~mm}$ path length.

\section{Dissociation Constants}

The dissociation constant of BPB in the polymerization system was estimated by the method of McInnes and Shedlovsky ${ }^{4}$ as follows.

Equivalent conductances $(\Lambda)$ of BPB were measured at several concentrations $(c)$ by Yanagimoto Conductivity Outfit Model MY-7. The limiting equivalent conductance $\left(\Lambda_{0}\right)$ was obtained by extrapolating the linear plot of $\Lambda$ versus $c^{1 / 2}$ to $c=0$. Apparent degree of dissociation $\left(\alpha^{*}\right)$ and dissociation constant $\left(K^{*}\right)$ of BPB were respectively calculated by the following equation.

$$
\begin{aligned}
& \alpha^{*}=\Lambda / \Lambda_{0} \\
& K^{*}=\alpha^{* 2} c / 1-\alpha^{*}
\end{aligned}
$$

The actual dissociation constant $(K)$ was estimated by the extrapolation of the $\log K^{*}$ versus $\left(\alpha^{*} c\right)^{1 / 2}$ plot to the intercept. The actual degree of dissociation $(\alpha)$ at a concentration $(c)$ was calculated by the following equation.

$$
K=\alpha^{2} c / 1-\alpha
$$

\section{Photopolymerization}

Monomer, solvent and BPB were placed in a hard glass ampoule, which was degassed and substituted with nitrogen, and then sealed under vacuum. The ampoule was set on the rotating drum $5 \mathrm{~cm}$ from each of three Toshiba Fluorescent Chemical Lamps FL20SBL (20W, $\left.\lambda_{\max }=360 \mathrm{~nm}\right)$ and irradiated at $303 \pm 0.5 \mathrm{~K}$. The contents of the ampoule were poured into methanol and the precipitated polymer was dried in vacuo. In the case of photopolymerization in methylene chloride, no precipitate was obtained, and thus all the contents of the ampoule were evaporated in vacuo to dryness. Photocopolymerization of St and MMA was carried out by the same procedure. The composition of the copolymer was determined from absorbance at $260 \mathrm{~nm}$ in methylene chloride.

\section{Molecular Weight}

The Molecular weight of PMMA was determined by gel permeation chromatography (GPC) and viscometry.

GPC experiments were carried out on a Yanagimoto High Performance Chromatograph Model L-2000, equipped with a column Shodex A802-A803, using tetrahydrofuran as the eluting solvent. The molecular weight of PMMA was estimated from the elution volume of the polymer and compared with that of a standard polystyrene.

The intrinsic viscosity of PMMA was measured with an Ubbelohde dilution viscometer in benzene at $303 \mathrm{~K}$, and its molecular weight was calculated by the following equation. ${ }^{5}$

$$
[\eta]=8.69 \times 10^{-5} M_{n}^{0.76}
$$

\section{RESULTS AND DISCUSSION}

\section{Photopolymerization of $M M A$ with $B P B$}

Photopolymerization of MMA with BPB was carried out in three different solvents, methylene chloride, acetonitrile and methanol. In the case of methylene chloride and acetonitrile, there was no precipitate on the addition of methanol to the polymerization system, but small quantities of brownish powder remained following evaporation of the unreacted monomer and solvent. However, in the photopolymerization in methanol, PMMA was precipitated by the addition of methanol in large quantities. The conversion-time relations of these polymerizations are shown in Figure 1 indicate that

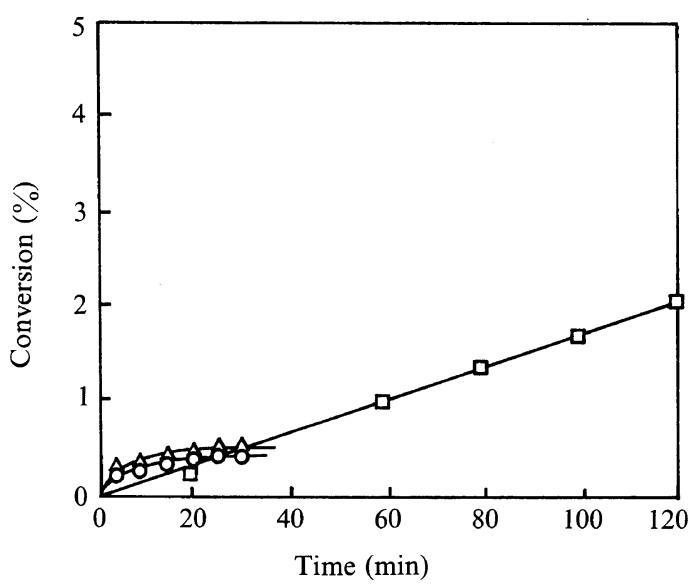

Figure 1. Conversion-time relation for the photopolymerization of MMA with BPB in different solvents: $\mathrm{BPB}, 1 \times 10^{-3} \mathrm{moldm}^{-3}$; MMA, $5 \mathrm{~cm}^{3}$; solvent, $5 \mathrm{~cm}^{3}$; $\mathrm{O}, \mathrm{CH}_{2} \mathrm{Cl}_{2} ; \triangle, \mathrm{CH}_{3} \mathrm{CN} ; \square, \mathrm{CH}_{3} \mathrm{OH}$. 


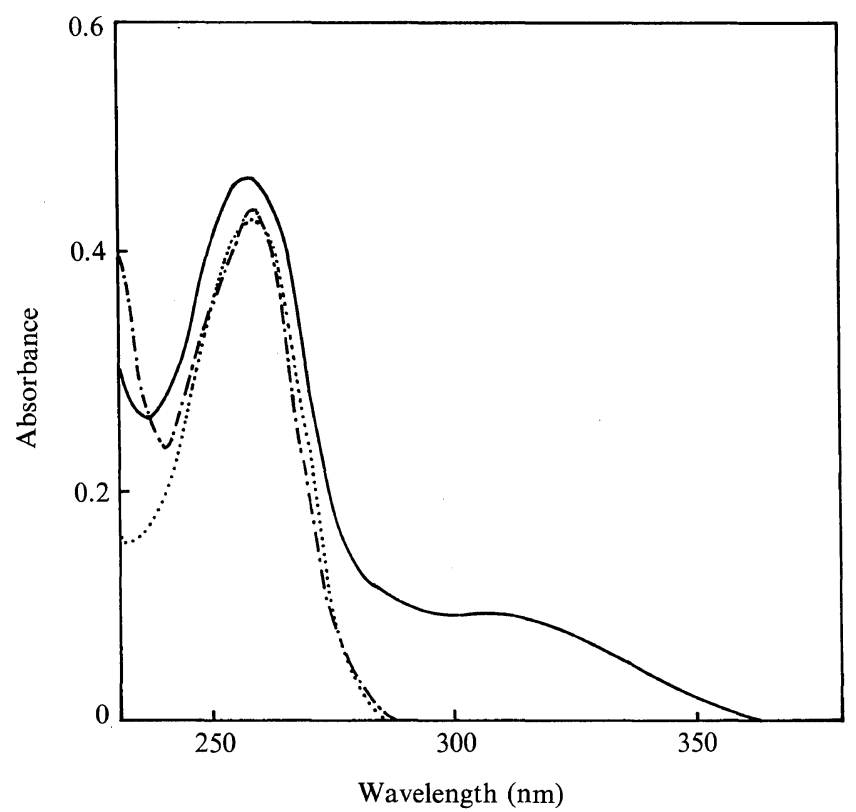

Figure 2. UV spectra of $\mathrm{BPB}\left(1 \times 10^{-4} \mathrm{~mol} \mathrm{dm}^{-3}\right)$ : - - in $\mathrm{CH}_{2} \mathrm{Cl}_{2} ;-\cdot-\cdot-$, in $\mathrm{CH}_{3} \mathrm{CN} ; \cdots \cdot$, in $\mathrm{CH}_{3} \mathrm{OH}$.

the conversion did not exceed over $1 \%$ in either methylene chloride or acetonitrile, but that in methanol, a linear conversion-time relation was found over a wide range of conversion. The molecular weights of PMMA obtained in methylene chloride and acetonitrile were estimated by GPC as 4,400 and 14,000 , respectively. The molecular weight of PMMA obtained in methanol was estimated as 580,000 by viscometry.

In the previous paper, ${ }^{2}$ the authors assumed that the photopolymerization of MMA with BPPB in methanol was initiated by radicals formed by the photodecomposition of the charge transfer complex induced from BPPB. Ghosh and Mitra also examined the effectiveness of quinoline-bromine $\left(\mathrm{Q}-\mathrm{Br}_{2}\right)$ and pyridine-bromine $\left(\mathrm{Py}-\mathrm{Br}_{2}\right)$ charge transfer complexes as photosensitizers of vinyl polymerization. ${ }^{6,7}$ They suggest that the polymerization is initiated by a bromine atom produced from the decomposition of $\mathrm{Q}-\mathrm{Br}_{2}$ and $\mathrm{Py}-\mathrm{Br}_{2} \mathrm{CT}$ complexes and is terminated according to the bimolecular termination mechanism.

The UV spectra of BPB measured in methanol, acetonitrile and methylene chloride are shown in Figure 2. All the spectra show large pyridinium ring absorption at $258 \mathrm{~nm}$. The spectrum in methylene

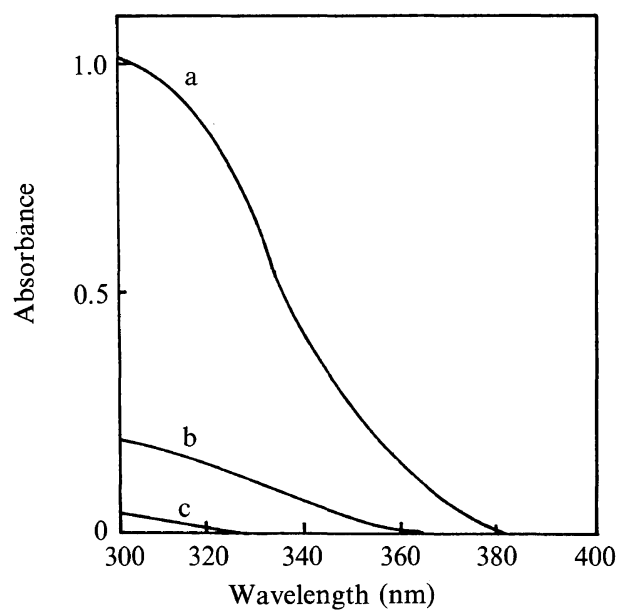

Figure 3. UV spectra of BPB in MMA-solvent mixtures: BPB, $1 \times 10^{-3} \mathrm{~mol} \mathrm{dm}^{-3}$; (a), $\mathrm{MMA}-\mathrm{CH}_{2} \mathrm{Cl}_{2}$ (1:1); (b), MMA- $\mathrm{CH}_{3} \mathrm{CN}$ (1:1); (c), MMA- $\mathrm{CH}_{3} \mathrm{OH}$ $(1: 1)$.

chloride, however, show another absorption band in the wavelength region from 290 to $350 \mathrm{~nm}$, which may possibly be the CT absorption of BPB according to the analogy of the spectrum of pyridinium iodide reported by Kosower. ${ }^{8}$ The UV spectra of 


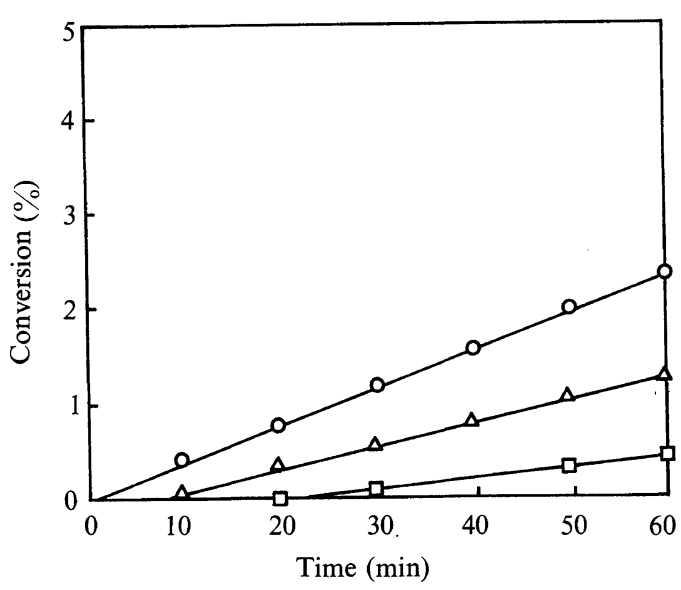

Figure 4. Conversion-time relations for the photopolymerization of MMA with $\mathrm{BPB}$ in methanol in the presence of TEAB: MMA, $5 \mathrm{~cm}^{3} ; O,[\mathrm{BPB}]=1 \times 10^{-3}$ mol dm ${ }^{-3},[\mathrm{TEAB}]=1 \times 10^{-2} \mathrm{~mol} \mathrm{dm}^{-3} ; \triangle,[\mathrm{BPB}]=$ $1 \times 10^{-3} \mathrm{~mol} \mathrm{dm}^{-3} ; \square,[\mathrm{TEAB}]=1 \times 10^{-2} \mathrm{~mol} \mathrm{dm}^{-3}$.

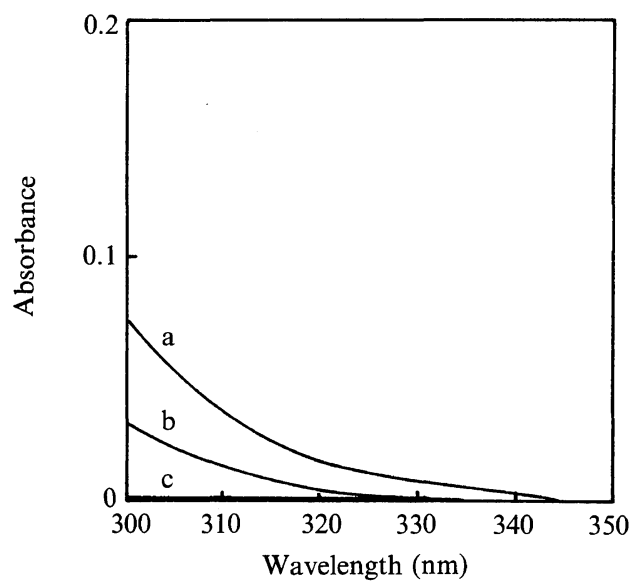

Figure 5. UV spectra of $\mathrm{BPB}$ in $\mathrm{MMA}-\mathrm{CH}_{3} \mathrm{OH}(1: 1)$ in the presence of TEAB: (a), $[\mathrm{BPB}]=1 \times 10^{-3}$ mol dm ${ }^{-3},[\mathrm{TEAB}]=1 \times 10^{-2} \mathrm{~mol} \mathrm{dm}^{-3} ;(\mathrm{b}),[\mathrm{BPB}]=$ $1 \times 10^{-3} \mathrm{~mol} \mathrm{dm}^{-3}$; (c), $\left[\right.$ TEAB] $=1 \times 10^{-2} \mathrm{~mol} \mathrm{dm}^{-3}$.

BPB were also measured in these solvents each containing an equal volume of MMA (Figure 3). The results show that the intensity of the CT band decrease in proportion to the polarity of the solvent increased. The dissociation constants $(K)$ of BPB were also determined in an MMA-solvent $(1: 1)$. The values obtained in $\mathrm{MMA}-\mathrm{CH}_{3} \mathrm{OH}(1: 1)$ and MMA- $\mathrm{CH}_{3} \mathrm{CN} \quad(1: 1)$ were $4.88 \times 10^{-4}$ and $5.37 \times 10^{-4} \mathrm{~mol} \mathrm{dm}^{-3}$, respectively. BPB in MMA-

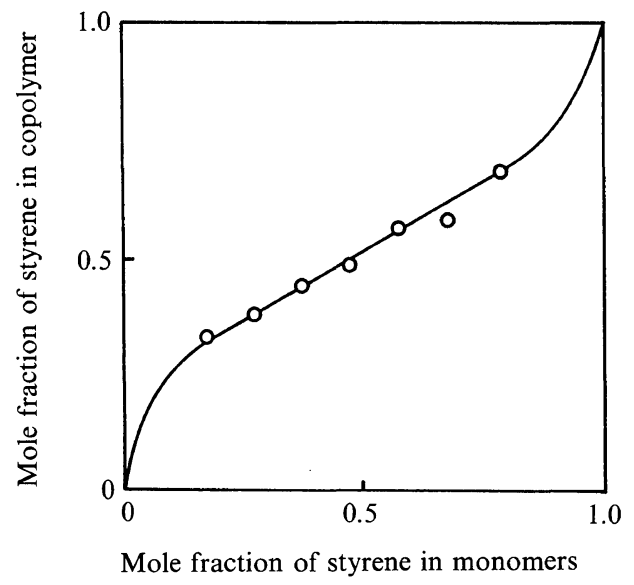

Figure 6. Composition curve in the copolymerization of styrene and MMA: styrene, $2.5 \mathrm{~cm}^{3}$; MMA, $2.5 \mathrm{~cm}^{3}$; methanol, $5 \mathrm{~cm}^{3}$; BPB, $1 \times 10^{-3} \mathrm{~mol} \mathrm{dm}^{-3}$; temp, $303 \mathrm{~K}$; time, $1 \mathrm{~h}$.

$\mathrm{CH}_{2} \mathrm{Cl}_{2}$ (1:1), however, showed no electric conductivity. From these results, it is considered that in MMA- $\mathrm{CH}_{3} \mathrm{OH}$ and MMA- $\mathrm{CH}_{3} \mathrm{CN}$, BPB dissociated into free ions, $N$-benzylpyridinium and bromide ions.

The photopolymerization of MMA with BPB was carried out in methanol in the presence of TEAB ten times more concentrated than BPB. Figure 4 shows that the photopolymerization was accelerated by the addition of TEAB. UV spectra were also measured in the same polymerization systems as described above. The intensity of the CT absorption distinctly increased with the addition of TEAB (Figure 5). These facts imply that there exists an equilibrium among free ions, and ion pair $^{9}$ and the CT complex of BPB as shown in eq 1. It is also considered that the equilibrium shifts to the right with an increase in the $\mathrm{Br}^{-}$concentration derived from TEAB, and that the CT complex of BPB serves as a photosensitive species for the polymerization.

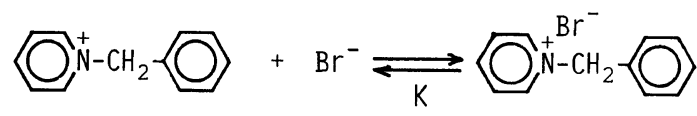

Free ion Ion pair

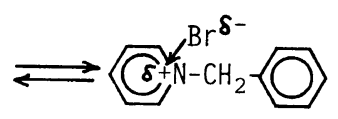




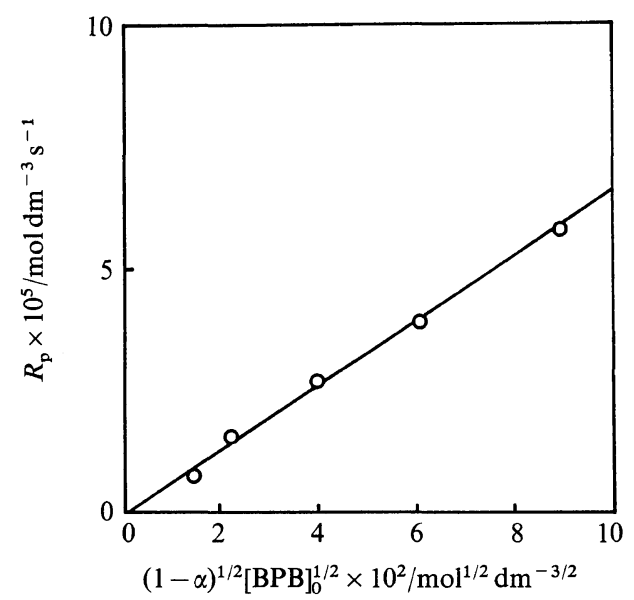

Figure 7. $R_{\mathrm{p}}$ versus $(1-\alpha)^{1 / 2}[\mathrm{BPB}]_{0}^{1 / 2}$ for the photopolymerization of MMA with BPB in methanol.

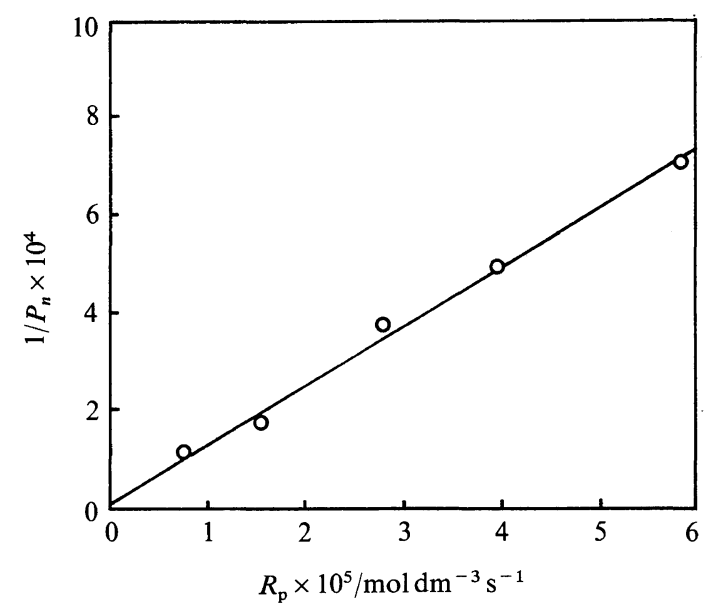

Figure 8. $1 / P_{n}$ versus $R_{\mathrm{p}}$ for the photopolymerization of MMA with BPB in methanol.

In order to confirm the radical mechanism for the photopolymerization, St and MMA were photocopolymerized with BPB in methanol. The composition curve of the copolymer is shown in Figure 6 , and corresponds to that of the radical polymerization. The initial rates $\left(R_{\mathrm{p}}\right)$ of the photopolymerization of MMA in methanol in the presence of various concentrations of $\mathrm{BPB}$ were plotted against $(1-\alpha)^{1 / 2}[\mathrm{BPB}]_{0}^{1 / 2}$, where $(1-\alpha)[\mathrm{BPB}]_{0}$ shows the initial concentration of undissociated BPB. A linear plot was obtained as shown in Figure 7. The

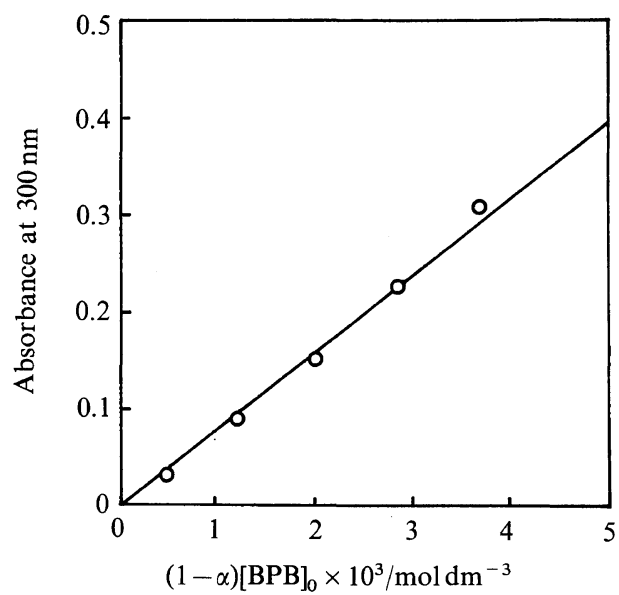

Figure 9. Absorbance at $300 \mathrm{~nm}$ versus $(1-\alpha)[\mathrm{BPB}]_{0}$ for CT absorption of BPB in MMA- $\mathrm{CH}_{3} \mathrm{OH}(1: 1)$.

relation between the reciprocal of the degree of polymerization $\left(1 / P_{n}\right)$ and $R_{\mathrm{p}}$ was also linear (Figure 8). These results accord with the fact that absorbances at $300 \mathrm{~nm}$ (CT band) in the same polymerization systems as used in kinetic studies were proportional to $(1-\alpha)[\mathrm{BPB}]_{0}$ (Figure 9). Therefore, the photopolymerization is probably initiated by a bromine atom, produced by the photodecomposition of the CT complex of BPB as shown in eq 2. $N$-Benzylpyridinyl radical was too stable ${ }^{10}$ to initiate the polymerization of MMA.

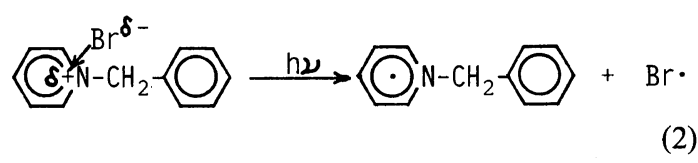

Photo-Oligomerization of MMA with BPB in Methylene Chloride

As above described, the photopolymerization of MMA with BPB in methylene chloride gave PMMA with a relatively low molecular weight, and thus it was expected that an OMMA could be obtained in methylene chloride at a higher concentration of BPB and lower concentration of MMA. In fact, the conversion of the photopolym-

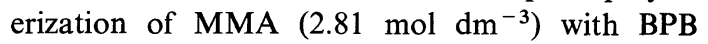
$\left(5 \times 10^{-3} \mathrm{~mol} \mathrm{dm}^{-3}\right)$ in methylene chloride was not able to exceed $0.7 \%$, and the molecular weight of the obtained OMMA was estimated to be 600 by GPC. This value is distinctly lower than that expected for the usual radical polymerization of 


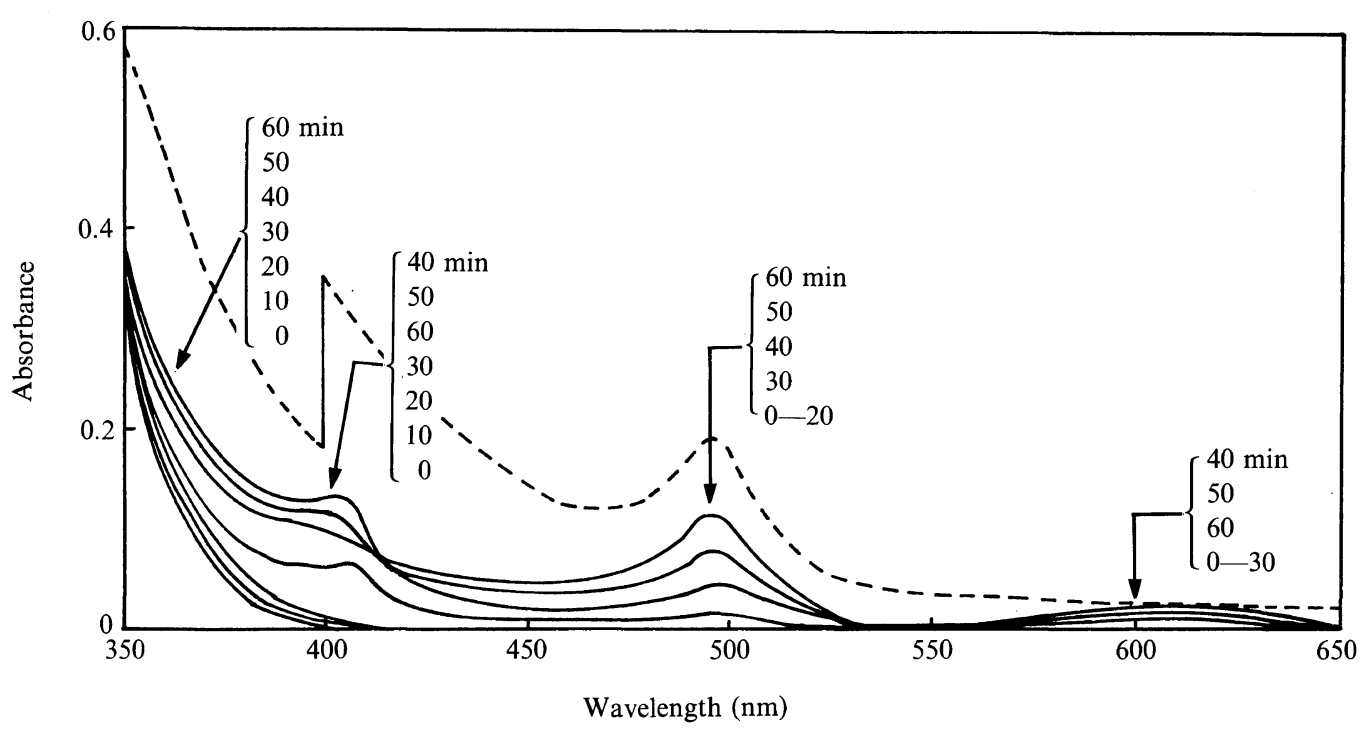

Figure 10. The spectral change in the reaction system with photo-oligomerization time. The dotted line shows the spectrum of freshly prepared $\mathrm{BPBH}$ in methylene chloride.

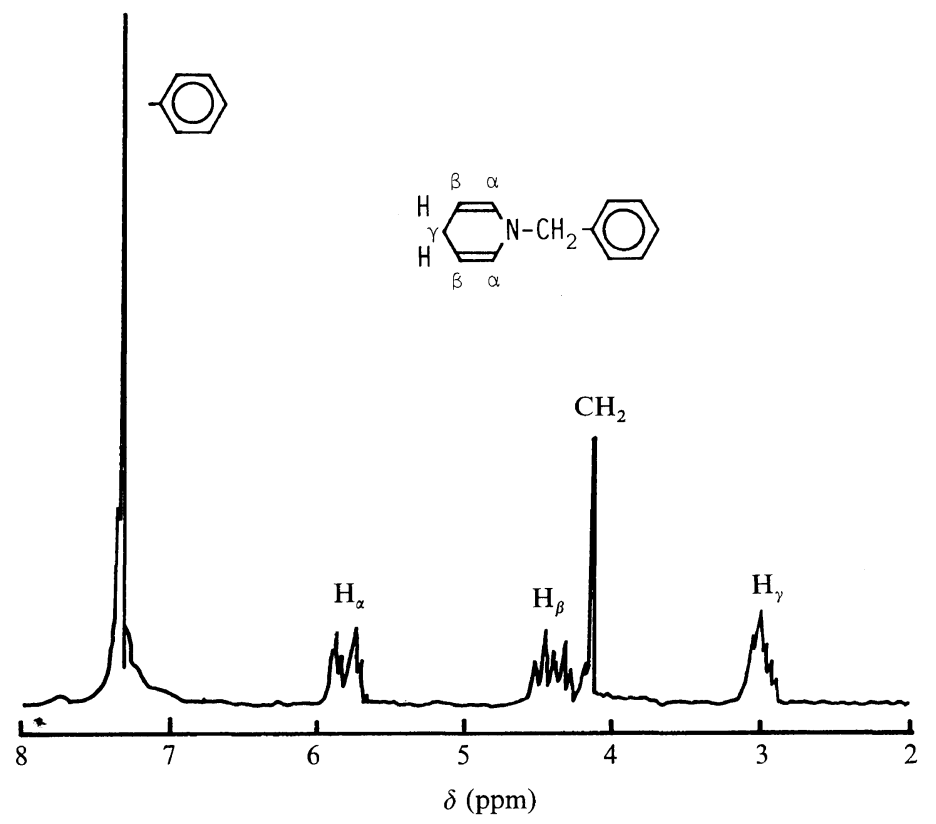

Figure 11. ${ }^{1} \mathrm{H}$ NMR spectrum of $\mathrm{BPBH}$ in $\mathrm{CDCl}_{3}$.

MMA.

The polymerization system in the ampoule was colorless prior to irradiation and became increasingly brownish with irradiation time. The spectral change of the system is shown in Figure 10. Absorption maxima appeared at 405, 495 and $620 \mathrm{~nm}$ with irradiation. The absorption maxima appeared at 405 and $620 \mathrm{~nm}$ but gradually disap- 


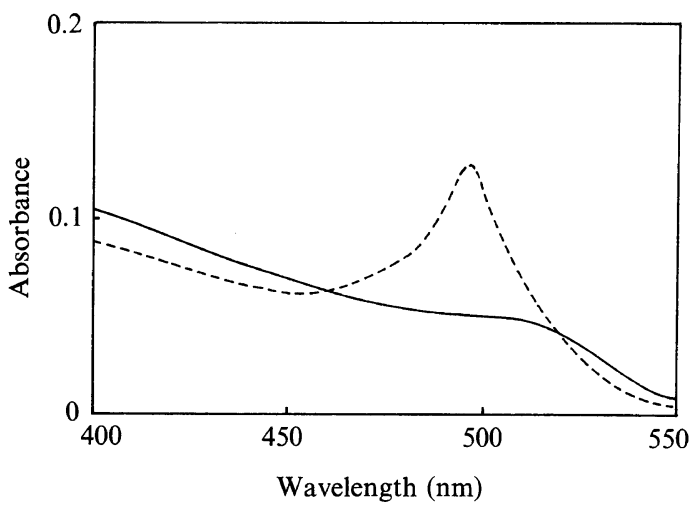

Figure 12. Absorption spectra (----), for the reaction system immediately following photo-oligomerization and (-), the residual OMMA in methylene chloride after removal of the unreacted MMA and solvent; concentration, arbitrary.

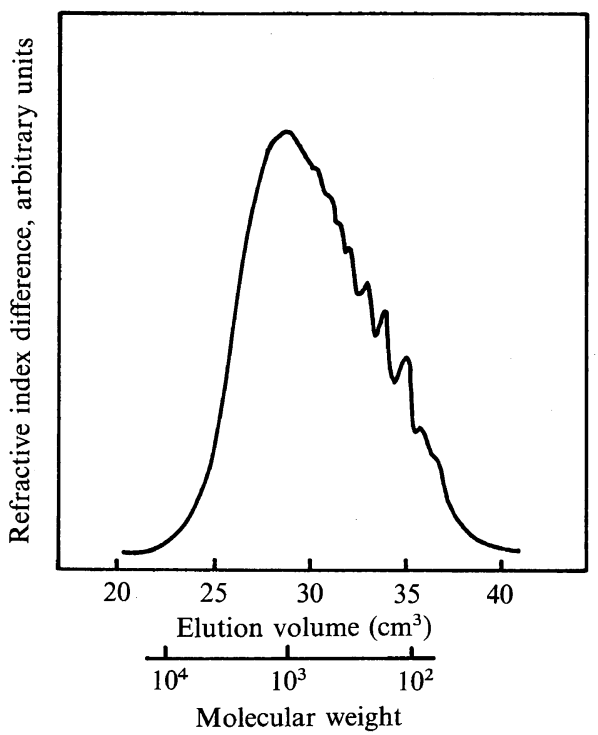

Figure 13. Gel permeation chromatogram for OMMA prepared by the photo-oligomerization of MMA with BPB in methylene chloride: MMA, $2.81 \mathrm{~mol} \mathrm{dm}^{-3}$; BPB, $5 \times 10^{-3} \mathrm{~mol} \mathrm{dm}^{-3}$; conversion, $0.75 \%$; GPC column, Shodex A802-A803; eluent, THF.

peared, the one at $495 \mathrm{~nm}$ continued to increase in size with irradiation time. These spectral changes during irradiation cannot be explained exactly, but the absorption spectrum of BPB following irradiation seems similar to that of 1-benzyl-1,4dihydropyridine (BPBH). A fresh preparation of
Table I. Bromine and nitrogen content of OMMA ${ }^{\mathrm{a}}$

\begin{tabular}{cccc}
\hline Bromine content & & Nitrogen content \\
\cline { 1 - 2 } $\begin{array}{r}\text { Average number of } / \% \text { bromine atoms per } \\
\text { OMMA molecule }\end{array}$ & & $\begin{array}{r}\text { Average number of } \\
\text { OMitrogen atoms per } \\
\text { OMMA molecule }\end{array}$ \\
\hline 11.7 & 0.95 & 1.88 & 0.87 \\
\hline${ }^{a} M_{n}=650$ (by VPO). & &
\end{tabular}

BPBH was made by the reduction of BPB with sodium dithionite whose electronic spectrum is shown by a dotted line in Figure 10. The ${ }^{1} \mathrm{H}$ NMR spectrum of BPBH is shown in Figure 11. After photopolymerization, the unreacted MMA and solvent were distilled off under vacuum, and the electronic spectrum of the residual oligomer was measured in methylene chloride, but no significant absorption band was found at $495 \mathrm{~nm}$ (Figure 12). After exposure to air, the absorption band at $495 \mathrm{~nm}$ of BPBH slowly disappeared and a new broad band appeard in the wave length region from 400 to $450 \mathrm{~nm}$. A possible explanation for these results is the instability of dihydropyridine derivatives, as suggested by Acheson and Paglietti. ${ }^{11}$

In order to obtain a large quantity of the oligomer for structural analysis, photo-oligomerization was performed under a nitrogen atmosphere using photochemical reaction apparatus with a Fuji Glass High Pressure Mercury Lamp HL-100 at the same concentration of MMA and BPB described before. The OMMA thus prepared was purified by chromatography on a silica gel column with acetone. By this method, any pyridinium salts and tetrahydrobispyridine were removed. Number average molecular weight $\left(M_{n}\right)$ of the purified OMMA was determined to be 650 by vapor pressure osmometry. The molecular weight distribution estimated by GPC is shown in Figure 13. The bromine and nitrogen content was determined by Konishi and Ishizuka's ${ }^{12}$ and Kjeldahl's methods respectively; the average number of bromine and nitrogen atoms contained in an oligomer molecule was calculated as 0.95 and 0.87 , respectively, and shown in Table I. From the results of ${ }^{1} \mathrm{H}$ and ${ }^{13} \mathrm{C}$ NMR measurements of the OMMA, the presence of these end groups could not be ascertained clearly. These results, however, suggest 
that the OMMA had structure $1(\bar{n}=4)$, and that the oligomerization proceeded by the mechanism shown in eq 2-5.

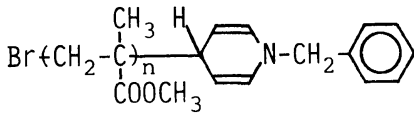

$$
\begin{aligned}
& 1 \\
& \mathrm{PyBr}(\mathrm{BPB}) \stackrel{h v}{\longrightarrow} \mathrm{Py} \cdot+\mathrm{Br} \text {. } \\
& \mathrm{Br} \cdot+\mathrm{M} \longrightarrow \mathrm{Br}-\mathrm{M}_{1} \text {. } \\
& \mathrm{Br}-\mathrm{M}_{n} \cdot+\mathrm{M} \longrightarrow \mathrm{Br}-\mathrm{M}_{n+1} \text {. } \\
& \mathrm{Br}-\mathrm{M}_{n} \cdot+\mathrm{Py} \cdot \longrightarrow \mathrm{Br}-\mathrm{M}_{n}-\mathrm{Py}
\end{aligned}
$$

The bromine atom initiated the polymerization of MMA (eq 3), whereas the $N$-benzylpyridinyl radical $(\mathrm{Py} \cdot)$ contributed to the termination rather than initiation due to the stability of the radical (eq 5). The rate of termination with Py - was comparable to that of the propagation (eq 4), and so an oligomer was probably formed. A similar mechanism is known for the polymerization of vinyl monomer with phenylazotriphenylmethane ${ }^{13}$ and aromatic diazothioether. ${ }^{14}$

On the other hand, the photopolymerization of MMA with BPB in methanol gave PMMA with a high molecular weight as mentioned in the preceding section. This seems attributable to the fast reaction between $\mathrm{Py} \cdot$ and methanol, as suggested by
Kosower et al. ${ }^{10}$ and Ikegami et al. ${ }^{15}$

\section{REFERENCES}

1. N. Sakota, T. Tanigaki, and K. Tabuchi, Kogyo Kagaku Zasshi, 72, 975 (1969).

2. K. Tabuchi, T. Okubo, T. Tanigaki, and N. Sakota, Kogyo Kagaku Zasshi, 74, 469 (1971).

3. K. Tabuchi, T. Okubo, T. Tanigaki, and N. Sakota, Nippon Kagaku Kaishi, 1561 (1982).

4. D. A. MacInnes and T. Shedlovsky, J. Am. Chem. Soc., 54, 1429 (1932).

5. T. G Fox, J. B. Kinsinger, H. F. Mason, and E. M. Shule, Polymer, 3, 71 (1962).

6. P. Ghosh and P. S. Mitra, J. Polym. Sci., Polym. Chem. Ed., 13, 921 (1975).

7. P. Ghosh and P. S. Mitra, J. Polym. Sci., Polym. Chem. Ed., 14, 981 (1976).

8. E. M. Kosower, J. Am. Chem. Soc., 80, 3253 (1958).

9. "Ion pair" is "solvent-separated ion pair" as described by Kosower. E. M. Kosower, J. Am. Chem. Soc., 80, 3267 (1958).

10. E. M. Kosower and E. J. Poziomek, J. Am. Chem. Soc., 86, 5515 (1964).

11. R. M. Acheson and G. Paglietti, J. Chem. Soc., Perkin Trans. 1, 45 (1976).

12. H. Konishi and T. Ishizuka, Kobunshi Kagaku, 17, 169 (1960).

13. G. S. Misra, A. Hafeez, and K. S. Sharm, Makromol. Chem., 51, 123 (1962).

14. S. Niino, K. Ageishi, M. Takeishi, and S. Hayama, Kobunshi Ronbunshu, 35, 649 (1978).

15. Y. Ikegami and H. Watanabe, Chem. Lett., 1007 (1976). 